International Journal of Biomedicine | June 2019 - Volume 9, Issue Suppl_1: Abstracts From the Second Russian International Conference "Cryo-electron microscopy 2019: achievements and prospects"

POSTER ABSTRACT PRESENTATIONS

SESSION TITLE: MOLECULAR ORGANIZATION OF CELLS AND ORGANELLES

DOI: 10.21103/IJBM.9.Suppl_1.P27

\title{
Abstract P-27: Computational Modeling of Tubulin Protofilament Relaxation during Rapid Freezing
}

\author{
Dmitrii S. Vinogradov ${ }^{1}$, Evgeniy V. Ulyanov ${ }^{2}$, J. Richard McIntosh ${ }^{3}$, \\ Fazoil I. Ataullakhanov ${ }^{1,2}$, Nikita B. Gudimchuk ${ }^{1,2}$ \\ ${ }^{1}$ Center for theoretical problems of physicochemical pharmacology, Moscow, Russia $;{ }^{2}$ Physics \\ Department, Lomonosov Moscow State University, Moscow, Russia; ${ }^{3}$ MCD Biology Department, \\ University of Colorado, Boulder, CO, USA
}

Background: Over the recent years, there has been a burst of ground-breaking studies, based on cryo-EM. They shed light on various intracellular structures, including microtubules (Alushin et al., 2014; Manka and Moores, 2018). Composed of 13 laterally stacked tubulin protofilaments, microtubules can exist in growth and shortening states, enabling them to search and capture chromosomes in mitosis. In our recent cryo-electron tomography study, we directly examined the shapes of protofilaments at the microtubule tips (McIntosh et al., 2018). We discovered an unexpected feature of tubulin protofilaments: they were highly curved at the tip, but progressively less curved as one moved closer to the microtubule lattice.

Methods: We have hypothesized that the gradient of curvature in protofilaments could be explained by their incomplete relaxation during rapid freezing. To test this idea, here we use Brownian dynamics method (Ermak and McCammon, 1978). Protofilament is described as longitudinally bounded spheres, approximating tubulin monomers that can move only in the plane containing microtubule axis. Cooling rates achieve $10^{6}-10^{7} \mathrm{~K} / \mathrm{s}$ (Dubochet et al., 1988). Water viscosity increases in response to temperature drop according to published data (Dehaoui et al., 2015).

Results: We simulated fast freezing of soft and rigid protofilaments, having flexural stiffness coefficients of 35 and $174 \mathrm{kcal} \cdot \mathrm{mol}^{-1} \mathrm{rad}^{-2}$, respectively. At $10^{6} \mathrm{~K} / \mathrm{s}$ freezing rate both soft and rigid protofilaments had enough time to relax before cryo-immobilization, so that no gradient of curvature was predicted. When cooled at $10^{7} \mathrm{~K} / \mathrm{s}$, rigid protofilaments again had enough time to fully relax. However, soft protofilaments displayed a gradient of curvature, very similar to our experimental data. This suggests that in principle fast freezing could cause the gradient of curvature. 

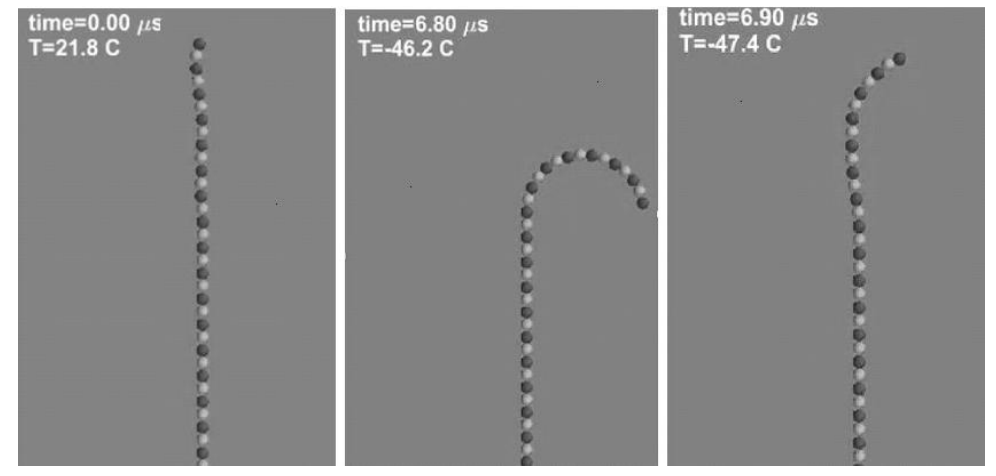

Fig. 1. Snapshots from protofilament freezing simulation. From left to right: initial straight state of protofilament, frozen protofilament with high flexural rigidity and frozen protofilament with low flexural rigidity. Cooling rate is $10^{7} \mathrm{~K} / \mathrm{s}$.

Conclusion: Due to fast cooling, configurations of some large biomolecules, captured by cryo-EM, may represent snapshots of their incompletely relaxed states. Thus, additional using Brownian dynamics method can be helpful to correctly interpret cryo-EM data.

Key Words: microtubules $\bullet$ tubulin $\bullet$ cryo-electron microscopy $\bullet$ freezing

Sources of Funding: This work was supported by Russian Science Foundation grant \#17-74-20152 to N.G. The research was carried out using the equipment of the shared research facilities of HPC computing resources at Lomonosov Moscow State University.

International Journal of Biomedicine. 2019;9 Suppl 1: S28-29. doi: 10.21103/IJBM.9.Suppl_1.P27

(C)2019 International Medical Research and Development Corporation 